\title{
Dental and Cranial Variation in Living Indriidae
}

\author{
Philip D. Gingerich and Alan S. Ryan \\ The University of Michigan
}

\begin{abstract}
Four species of Indriidae are extant in Madagascar. We have studied large samples of each of these to characterize dental and cranial variation, and to estimate the degree of sexual dimorphism in the dentition and cranium. Two dental fields are apparent, characterized by reduced variability: (1) a canine field centered on the upper canine and occluding caniniform lower premolar, and (2) a cheek tooth field centered on the second molars. No consistent pattern of sexual dimorphism was found in dental or cranial dimensions, and we conclude that none of the four species is sexually dimorphic. This lack of dental and cranial dimorphism is unusual in primates, and probably reflects the relatively limited aggressive behavior and the lack of male dominance in Indriidae.
\end{abstract}

\section{INTRODUCTION}

There are abundant data available on tooth size and patterns of dental variation in many species of anthropoid primates (SCHUMAN \& BRACE, 1954; FRISCH, 1963; Biggerstaff, 1966; Pilbeam, 1969; Leutenegger, 1971; Gingerich, 1974; JohanSON, 1974; SwINDLER, 1976; and others). The only source of data on tooth size and variation in prosimian primates is the recent book by SWINDLER (1976), where measurements are summarized for 12 species of prosimians. In several cases these data are based on very small samples, a problem that is especially serious for the only three species of Lemuroidea published by SwINDLER: Varecia variegata, Lepilemur mustelinus, and Propithecus verreauxi. A knowledge of patterns of variation in living prosimians is necessary for understanding morphological and functional fields in the dentition, and it is important also for interpreting paleontological samples in the fossil record. Our primary objective here is to describe tooth size and dental variation in four extant prosimian species, all of the family of Indriidae.

A second objective of the present study is to determine if there is any consistent pattern of sexual dimorphism in the dentitions or crania of Indriidae. Anthropoid primates are characterized by marked sexual dimorphism in which the males are generally larger in body size and have relatively larger canine teeth than conspecific females (Leutenegger \& Kelly, 1977). Prosimians, on the other hand, are usually said to lack sexual dimorphism in body size and in size of the canine teeth, although very little quantitative data has been published to support this. LEUTENEGGER (1973) has shown that Perodicticus and Arctocebus lack sexual dimorphism in dimensions of the pelvis. SwINDLER (1976) demonstrates a pattern of canine size dimorphism in Lorisoidea, and suggests that molar size is sexually dimorphic in Propithecus verreauxi.

The family Indriidae is confined to Madagascar, and it includes four living species: 
Avahi laniger, Propithecus verreauxi, $P$. diadema, and Indri indri. These four species form a fairly homogeneous group morphologically, although they range in body weight from about $1.3 \mathrm{~kg}$ in Avahi to $3.5 \mathrm{~kg}$ in Propithecus, and $6.3 \mathrm{~kg}$ in Indri (BAUCHOT \& STEPHAN, 1966). All are vertical clingers and leapers, all are arboreal leafeaters, and all live in small family groups usually lacking male dominance and distinguished by limited intraspecific aggression (PETTER, 1962; NAPIER \& NAPIER, 1967; RICHARD, 1974; Pollock, 1975). Avahi laniger is distributed along the northwestern and eastern coasts of Madagascar, Propithecus verreauxi inhabits the western and southern coasts of Madagascar, while Propithecus diadema and Indri indri are confined to the northern and eastern regions of the country.

Seven recently extinct subfossil species are closely related to living Indriidae: Mesopropithecus pithecoides, $M$. globiceps, Palaeopropithecus ingens, Archaeoindris fontoynonti, Archaeolemur majori, A. edwardsi, and Hadropithecus stenognathus (TATTERSALl, 1973b). Data on dental and cranial variation in Mesopropithecus and in the latter three subfossil species are given by TATTERSALL $(1971,1973 \mathrm{a}$, respectively).

\section{MATERIAL AND METHODS}

This project was initiated as a study of dental variation in Indri indri. Four of the largest collections of Indri skulls and skeletons are housed in the Cleveland Museum of Natural History (U.S.A.); the British Museum of Natural History, London (England); the Rijksmuseum van Natuurlijke Historie, Leiden (Netherlands); and the Laboratoire d'Anatomie Compareé, Muséum National d'Histoire Naturelle, Paris (France). The osteological collection of Indri was studied in all four of these institutions, and the collections of Propithecus and Avahi were studied in the latter three. A fifth large systematic collection, in the American Museum of Natural History, New York (U.S.A.), was not studied because an initial inquiry indicated that most specimens of Indri were scattered on loan to different individuals and institutions.

Large samples of all four indriid species were studied: Avahi laniger ( 34 specimens), Propithecus verreauxi (63 specimens), Propithecus diadema (46 specimens), and Indri indri (56 specimens). Identification of the sex of each specimen, where known, was taken from original labels, and checked in museum or published catalogues (e.g., JeNTINK, 1887). Abbreviations used in the tables are: $n=$ sample size, $\overline{\mathrm{x}}=$ mean, $\mathrm{s}=$ standard deviation, $\mathrm{V}=$ coefficient of variation. The criterion of significance used in $t$-tests of sex differences was $p \leq .05$. All measurements were made in millimeters to the nearest $0.1 \mathrm{~mm}$, using a Helios dial caliper with specially sharpened points to facilitate mesial-distal measurements. Dental and cranial measurements were taken as illustrated in Figure 1. All measurements were made by the senior author; the junior author is responsible for the statistical computations.

Some comments are in order concerning tooth homologies in Indriidae. The dental formula of living indriids is usually given as $\frac{2}{1} \frac{1}{2} \frac{2}{2} \frac{3}{3}$, where the homologies of the upper teeth are understood to be $\mathrm{I}^{1-2}, \mathrm{C}, \mathrm{P}^{3-4}, \mathrm{M}^{1-3}$; and of the lower teeth $\mathrm{I}_{1-2}$, $P_{3-4}, M_{1-3}$. In recent papers, SchwarTz (1974) reinterpreted the anterior two teeth 

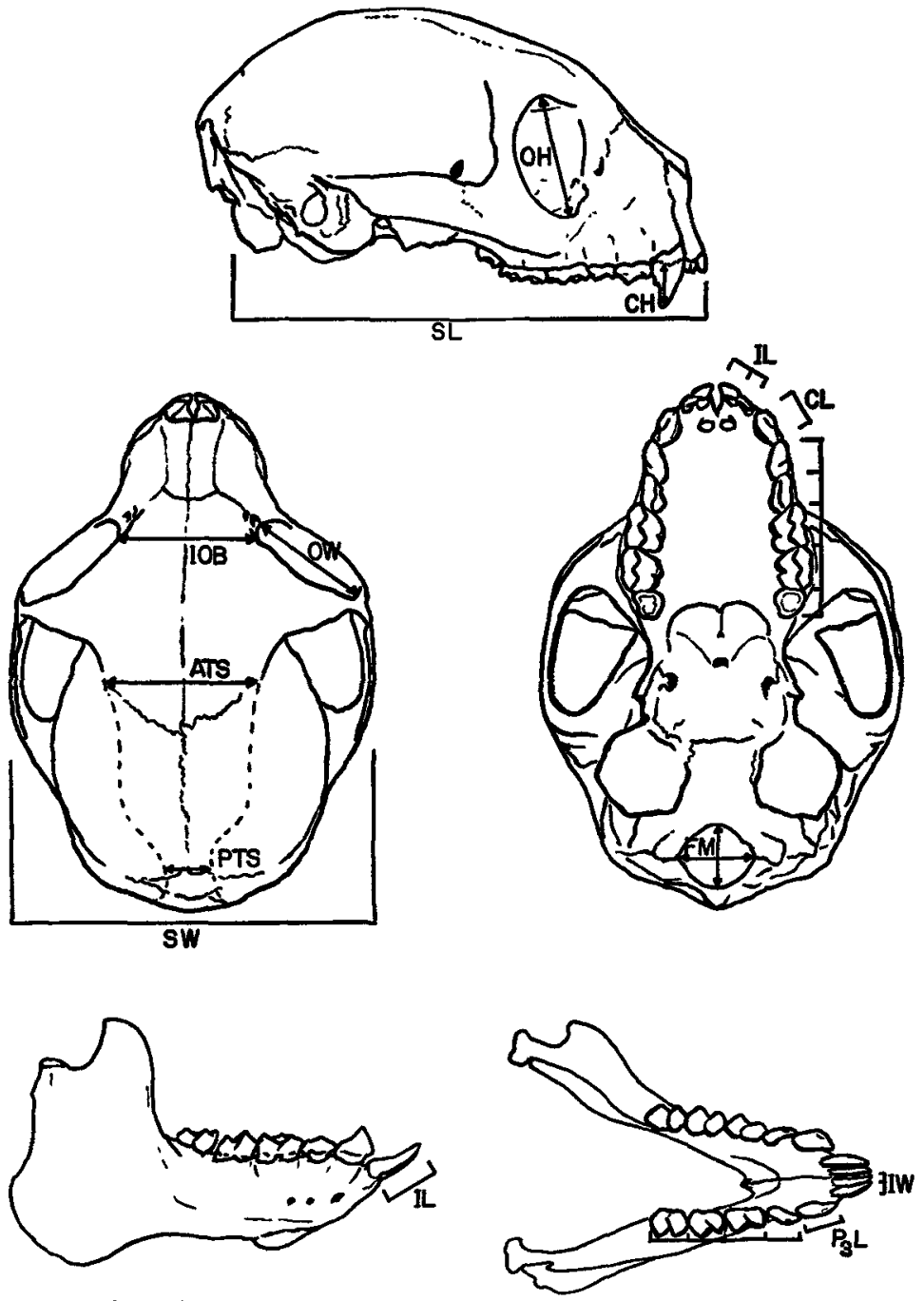

Fig. 1. Skull of Indri indri showing measurements of the dentition and cranium of Indriidae used in this study. Incisor length (IL) is crown length measured mesiodistally on upper incisors and anteroposteriorly on lowers. Incisor width (IW) is crown width measured labiolingually on uppers and mesiodistally on lowers. Canine length (CL) measured as shown, canine width perpendicular to this in occlusal plane, canine height $(\mathrm{CH})$ is projection of enameled crown on unworn or little worn teeth. Length of caniniform lower first premolar $\left(\mathbf{P}_{3}\right)$ measured as shown, crowns of other cheek teeth measured mesiodistally (length) and buccolingually (width). Cranial abbreviations: ATS, anterior temporal separation; FM, foramen magnum height and width; IOB, interorbital breadth; $\mathrm{OH}$, orbital height; $\mathrm{OW}$, orbital width; PTS, posterior temporal separation; SL, skull length; SW, skull width.

in the lower dentition as $I_{3}$ and $C$ rather than $I_{1}$ and $I_{2}$, and GoDFREY (1976) reinterpreted the lower premolars as $\mathbf{P}_{2}$ and $\mathbf{P}_{\mathbf{4}}$ rather than $\mathbf{P}_{\mathbf{3}}$ and $\mathbf{P}_{4}$. For reasons outlined elsewhere (GINGERICH, 1977), we think the evidence favors the traditional interpretation of the anterior lower teeth as $\mathbf{I}_{1}$ and $\mathbf{I}_{2}$ rather than ScHwarTz's 
proposed revision. On the other hand, the relative size of premolars in the deciduous dentition of indriids supports GoDFrEY's interpretation. We have followed the traditional system of numbering premolars here, but GODFREY may well be correct in proposing that what we have called $P_{3}$ is homologous with $P_{2}$ in generalized mammals.

\section{RESULTS}

The results of this study are given in Tables 1-11, with the more important patterns of variation being illustrated in Figures 2 and 3. Results of investigating basic dental variation, sexual dimorphism, cranial variation, and variation in tooth crown area are presented in that order.

\section{Dental Variation In INDRIIDAE}

Summary statistics for the length and width of each tooth in each of the four species of living Indriidae are given in Tables 1-4. The relative sizes of the teeth in all four species are nearly constant, and tooth size is in fact highly correlated with overall body size within the Indriidae.

There is significant variation in each of the measurements, but this variation is not completely random and it conforms to the general mammalian pattern. Coefficients of variation range from a low of 3.3 for $\mathrm{M}^{1}$ length in Indri indri $(n=51)$ to a high of 14.7 for $\mathrm{I}^{1}$ length in Avahi laniger $(n=18)$. This range in coefficients of variation is typical of that seen in other mammalian species (GINGERICH, 1974), although in highly sexually dimorphic species the coefficient can be much higher for canines and anterior premolars. Normally, the coefficients of variation for cheek teeth range from about three to about ten, and the values found here for indriid species are all within that range.

\section{SEXUAL DimORPHISM}

Dental measurements of the four species of Indriidae are separated by sex in Tables 5-8. T-tests were computed comparing means for measurements of males with those for conspecific females, and the measurements found to differ significantly $(p \leqq .05)$ are indicated by asterisks in a column on the left side in each table.

Males in our sample of Avahi laniger differ from females only in having a significantly wider $\mathrm{M}_{2}$ and $\mathrm{M}_{3}$. No significant differences in tooth size were found between males and females in our sample of Propithecus verreauxi. The upper canine in males of $P$. diadema is significantly wider than that of the females of this species in our sample, but otherwise the sexes are indistinguishable. Finally, the lengths of $\mathbf{M}^{2}$ and of $\mathrm{M}^{3}$ were the only significant differences in tooth size found in our sample of male and female Indri indri.

Several comments can be made about the statistical differences in tooth size found between males and females in Avahi laniger, Propithecus diadema, and Indri indri. First, there is no consistent pattern within the family, nor can differences in the length of $\mathrm{M}^{2-3}$ in one species, width of $\mathrm{M}^{2-3}$ in another, and width of the upper canine in a third be ascribed to any plausible functional difference. Secondly, with a 
Table 1. Dental measurements and summary statistics for all Avahi laniger specimens, with male, female, and specimens of unknown sex combined

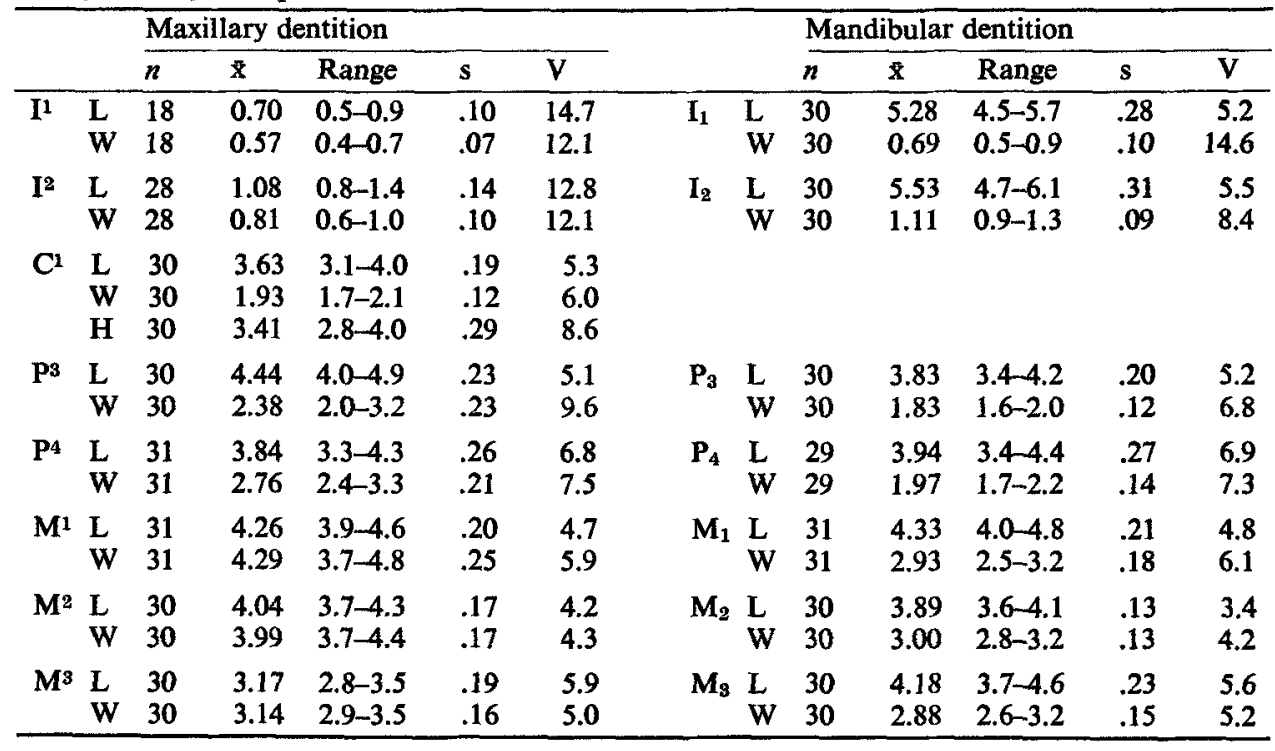

Table 2. Dental measurements and summary statistics for all Propithecus verreauxi specimens, with male, female, and specimens of unknown sex combined

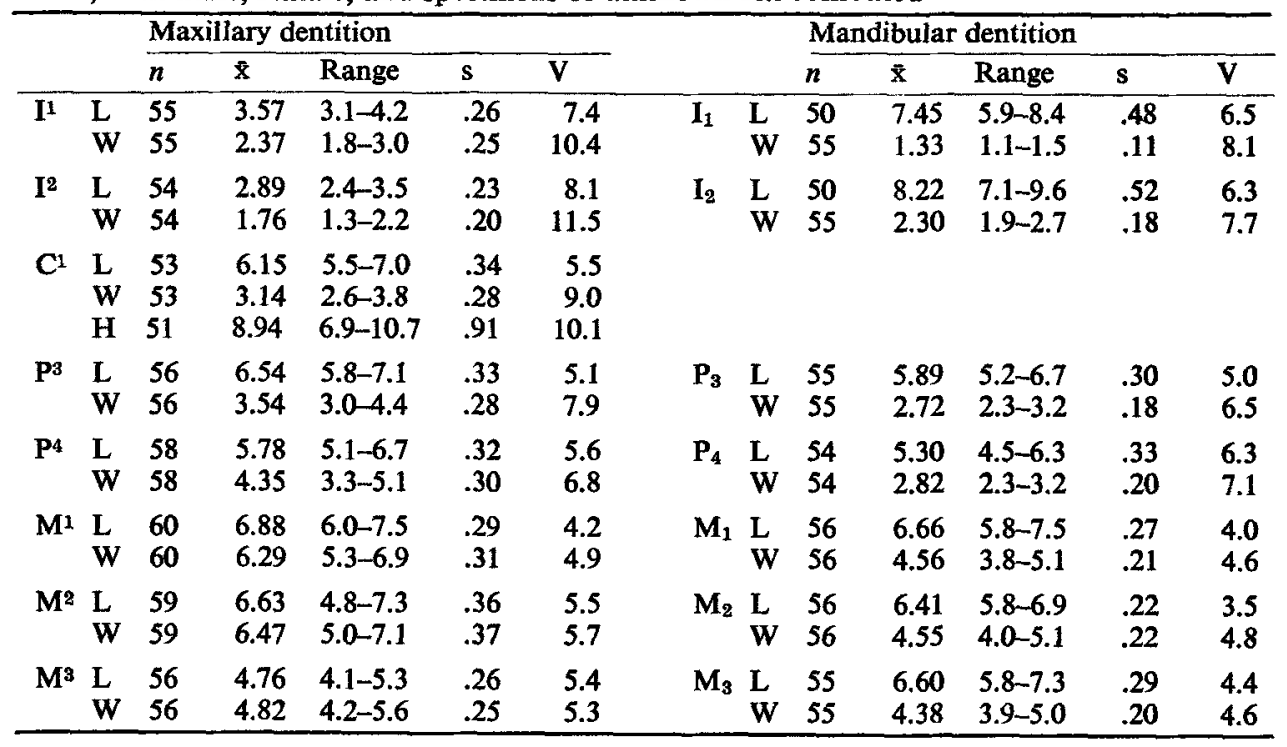

criterion of significance of $p=.05$, about one out of $20 t$-tests will appear significant by chance alone. Thirty-one measurements were taken on the teeth of each species, making a total of 124 measurements. Of these, two were significant at the .03 level, two were significant at the .02 level, and one was significant at the .01 level. The absence of a pattern in statistically significant differences, and the fact that only five 
Table 3. Dental measurements and summary statistics for all Propithecus diadema specimens, with male, female, and specimens of unknown sex combined

\begin{tabular}{|c|c|c|c|c|c|c|c|c|c|c|c|c|c|}
\hline & & \multicolumn{5}{|c|}{ Maxillary dentition } & & & \multicolumn{5}{|c|}{ Mandibular dentition } \\
\hline & & $n$ & $\overline{\mathbf{x}}$ & Range & $\mathrm{s}$ & V & & & $n$ & $\overline{\mathbf{x}}$ & Range & $s$ & V \\
\hline $\mathbf{I}^{1}$ & $\begin{array}{l}\mathbf{L} \\
\mathbf{W}\end{array}$ & $\begin{array}{l}42 \\
42\end{array}$ & $\begin{array}{l}.63 \\
3.01\end{array}$ & $\begin{array}{l}3.9-5.2 \\
2.5-3.4\end{array}$ & $\begin{array}{l}.33 \\
.25\end{array}$ & $\begin{array}{l}7.1 \\
8.2\end{array}$ & $I_{1}$ & $\begin{array}{l}\mathbf{L} \\
\mathbf{W}\end{array}$ & $\begin{array}{l}37 \\
38\end{array}$ & $\begin{array}{l}8.55 \\
1.58\end{array}$ & $\begin{array}{l}6.8-9.9 \\
1.3-1.9\end{array}$ & $\begin{array}{l}.71 \\
.17\end{array}$ & $\begin{array}{r}8.3 \\
11.0\end{array}$ \\
\hline$I^{2}$ & $\begin{array}{l}\mathrm{L} \\
\mathrm{W}\end{array}$ & $\begin{array}{l}42 \\
42\end{array}$ & $\begin{array}{l}3.23 \\
2.10\end{array}$ & $\begin{array}{l}2.6-3.7 \\
1.4-2.6\end{array}$ & $\begin{array}{l}.29 \\
.26\end{array}$ & $\begin{array}{r}9.1 \\
12.3\end{array}$ & $\mathbf{I}_{\mathbf{2}}$ & $\begin{array}{l}\mathbf{L} \\
\mathbf{W}\end{array}$ & $\begin{array}{l}40 \\
41\end{array}$ & $\begin{array}{l}9.44 \\
2.82\end{array}$ & $\begin{array}{l}8.0-10.8 \\
2.2-3.3\end{array}$ & $\begin{array}{l}.64 \\
.24\end{array}$ & $\begin{array}{l}6.8 \\
8.6\end{array}$ \\
\hline $\mathrm{C}^{\mathbf{1}}$ & $\begin{array}{l}\text { L } \\
\text { W } \\
\text { H }\end{array}$ & $\begin{array}{l}39 \\
39 \\
39\end{array}$ & $\begin{array}{l}6.12 \\
3.49 \\
9.77\end{array}$ & $\begin{array}{l}5.2-7.1 \\
2.9-4.0 \\
7.2-11.8\end{array}$ & $\begin{array}{r}.46 \\
.28 \\
1.03\end{array}$ & $\begin{array}{r}7.5 \\
7.9 \\
10.5\end{array}$ & & & & & & & \\
\hline $\mathbf{P}^{3}$ & $\begin{array}{l}\mathbf{L} \\
\mathbf{W}\end{array}$ & $\begin{array}{l}43 \\
43\end{array}$ & $\begin{array}{l}6.11 \\
3.85\end{array}$ & $\begin{array}{l}5.2-6.9 \\
3.3-4.9\end{array}$ & $\begin{array}{l}.36 \\
.35\end{array}$ & $\begin{array}{l}5.9 \\
9.1\end{array}$ & $\mathbf{P}_{3}$ & $\begin{array}{l}\mathbf{L} \\
\mathbf{W}\end{array}$ & $\begin{array}{l}40 \\
40\end{array}$ & $\begin{array}{l}5.87 \\
3.08\end{array}$ & $\begin{array}{l}5.2-6.7 \\
2.6-3.6\end{array}$ & $\begin{array}{l}.38 \\
.25\end{array}$ & $\begin{array}{l}6.4 \\
8.2\end{array}$ \\
\hline $\mathbf{P}^{4}$ & $\begin{array}{l}\text { L } \\
\text { W }\end{array}$ & $\begin{array}{l}44 \\
44\end{array}$ & $\begin{array}{l}5.63 \\
4.84\end{array}$ & $\begin{array}{l}4.6-7.5 \\
4.1-7.0\end{array}$ & $\begin{array}{l}.49 \\
.41\end{array}$ & $\begin{array}{l}8.8 \\
8.4\end{array}$ & $\mathbf{P}_{4}$ & $\begin{array}{l}\mathrm{L} \\
\mathrm{W}\end{array}$ & $\begin{array}{l}41 \\
41\end{array}$ & $\begin{array}{l}4.94 \\
3.15\end{array}$ & $\begin{array}{l}4.3-5.6 \\
2.4-3.7\end{array}$ & $\begin{array}{l}.32 \\
.26\end{array}$ & $\begin{array}{l}6.6 \\
8.2\end{array}$ \\
\hline $\mathbf{M}^{1}$ & $\begin{array}{l}\mathbf{L} \\
\mathbf{W}\end{array}$ & $\begin{array}{l}44 \\
44\end{array}$ & $\begin{array}{l}7.22 \\
7.01\end{array}$ & $\begin{array}{l}4.7-8.1 \\
4.0-7.9\end{array}$ & $\begin{array}{l}.53 \\
.62\end{array}$ & $\begin{array}{l}7.3 \\
8.8\end{array}$ & $\mathbf{M}_{\mathbf{1}}$ & $\begin{array}{l}\text { L } \\
\text { W }\end{array}$ & $\begin{array}{l}42 \\
42\end{array}$ & $\begin{array}{l}6.90 \\
5.00\end{array}$ & $\begin{array}{l}6.1-7.6 \\
4.4-5.7\end{array}$ & $\begin{array}{l}.37 \\
.29\end{array}$ & $\begin{array}{l}5.4 \\
5.7\end{array}$ \\
\hline $\mathbf{M}^{2}$ & $\begin{array}{l}\mathrm{L} \\
\mathbf{W}\end{array}$ & $\begin{array}{l}43 \\
43\end{array}$ & $\begin{array}{l}7.24 \\
7.31\end{array}$ & $\begin{array}{l}6.4-8.0 \\
6.4-8.3\end{array}$ & $\begin{array}{l}.39 \\
.42\end{array}$ & $\begin{array}{l}5.4 \\
5.8\end{array}$ & $\mathbf{M}_{2}$ & $\begin{array}{l}\mathbf{L} \\
\mathbf{W}\end{array}$ & $\begin{array}{l}42 \\
42\end{array}$ & $\begin{array}{l}7.08 \\
5.20\end{array}$ & $\begin{array}{l}6.4-7.7 \\
4.5-5.8\end{array}$ & $\begin{array}{l}.32 \\
.27\end{array}$ & $\begin{array}{l}4.5 \\
5.1\end{array}$ \\
\hline $\mathbf{M}^{3}$ & $\begin{array}{l}\mathrm{L} \\
\mathrm{W}\end{array}$ & $\begin{array}{l}41 \\
41 \\
\end{array}$ & $\begin{array}{l}5.68 \\
5.63 \\
\end{array}$ & $\begin{array}{l}4.9-6.6 \\
5.1-6.3 \\
\end{array}$ & $\begin{array}{l}.36 \\
.30 \\
\end{array}$ & $\begin{array}{l}6.4 \\
5.3 \\
\end{array}$ & $\mathbf{M}_{3}$ & $\begin{array}{l}\text { L } \\
\text { W }\end{array}$ & $\begin{array}{l}39 \\
39 \\
\end{array}$ & $\begin{array}{l}7.70 \\
5.20 \\
\end{array}$ & $\begin{array}{l}6.4-8.9 \\
4.5-5.9\end{array}$ & $\begin{array}{l}.47 \\
.30 \\
\end{array}$ & $\begin{array}{l}6.7 \\
5.7 \\
\end{array}$ \\
\hline
\end{tabular}

Table 4. Dental measurements and summary statistics for all Indri indri specimens, with male, female, and specimens of unknown sex combined

\begin{tabular}{|c|c|c|c|c|c|c|c|c|c|c|c|c|c|}
\hline & & \multicolumn{5}{|c|}{ Maxillary dentition } & & & \multicolumn{5}{|c|}{ Mandibular dentition } \\
\hline & & $n$ & $\overline{\mathbf{x}}$ & Range & $\mathbf{s}$ & $\mathrm{V}$ & & & $\bar{n}$ & $\overline{\bar{x}}$ & Range & $\mathrm{s}$ & $\mathrm{V}$ \\
\hline$I^{1}$ & $\begin{array}{l}\text { L } \\
\text { W }\end{array}$ & $\begin{array}{l}46 \\
47\end{array}$ & $\begin{array}{l}4.17 \\
2.10\end{array}$ & $\begin{array}{l}3.1-5.0 \\
1.8-2.6\end{array}$ & $\begin{array}{l}.43 \\
.17\end{array}$ & $\begin{array}{r}10.3 \\
8.2\end{array}$ & $\mathbf{I}_{1}$ & $\begin{array}{l}\text { L } \\
\text { W }\end{array}$ & $\begin{array}{l}48 \\
49\end{array}$ & $\begin{array}{r}10.76 \\
1.60\end{array}$ & $\begin{array}{l}9.0-11.9 \\
1.3-1.9\end{array}$ & $\begin{array}{l}.64 \\
.14\end{array}$ & $\begin{array}{l}5.9 \\
8.6\end{array}$ \\
\hline $\mathbf{I}^{2}$ & $\begin{array}{l}\text { L } \\
\text { W }\end{array}$ & $\begin{array}{l}47 \\
47\end{array}$ & $\begin{array}{l}4.43 \\
2.26\end{array}$ & $\begin{array}{l}3.3-5.1 \\
1.9-2.7\end{array}$ & $\begin{array}{l}.39 \\
.21\end{array}$ & $\begin{array}{l}8.8 \\
9.5\end{array}$ & $\mathbf{I}_{2}$ & $\begin{array}{l}\mathbf{L} \\
W\end{array}$ & $\begin{array}{l}48 \\
49\end{array}$ & $\begin{array}{r}11.37 \\
2.67\end{array}$ & $\begin{array}{c}10.1-12.6 \\
2.2-3.0\end{array}$ & $\begin{array}{l}.55 \\
.18\end{array}$ & $\begin{array}{l}4.8 \\
6.7\end{array}$ \\
\hline $\mathrm{C}^{1}$ & $\begin{array}{l}\text { L } \\
\text { W } \\
\text { H }\end{array}$ & $\begin{array}{l}51 \\
51 \\
51\end{array}$ & $\begin{array}{l}7.00 \\
3.83 \\
7.24\end{array}$ & $\begin{array}{l}6.3-7.8 \\
3.4-4.5 \\
5.9-8.4\end{array}$ & $\begin{array}{l}.39 \\
.25 \\
.60\end{array}$ & $\begin{array}{l}5.6 \\
6.5 \\
8.3\end{array}$ & & & & & & & \\
\hline P3 & $\begin{array}{l}\text { L } \\
\text { W }\end{array}$ & $\begin{array}{l}51 \\
51\end{array}$ & $\begin{array}{l}7.50 \\
4.43\end{array}$ & $\begin{array}{l}6.6-8.7 \\
3.7-5.4\end{array}$ & $\begin{array}{l}.39 \\
.38\end{array}$ & $\begin{array}{l}8.6 \\
5.2\end{array}$ & $\mathbf{P}_{3}$ & $\begin{array}{l}\mathrm{L} \\
\mathrm{W}\end{array}$ & $\begin{array}{l}50 \\
50\end{array}$ & $\begin{array}{l}6.93 \\
3.58\end{array}$ & $\begin{array}{l}5.9-7.7 \\
3.1-4.1\end{array}$ & $\begin{array}{l}.38 \\
.22\end{array}$ & $\begin{array}{l}5.5 \\
6.3\end{array}$ \\
\hline $\mathrm{p}^{4}$ & $\begin{array}{l}\mathbf{L} \\
\mathbf{W}\end{array}$ & $\begin{array}{l}51 \\
51\end{array}$ & $\begin{array}{l}6.90 \\
5.62\end{array}$ & $\begin{array}{l}5.9-8.0 \\
4.9-6.4\end{array}$ & $\begin{array}{l}.39 \\
.37\end{array}$ & $\begin{array}{l}5.7 \\
6.6\end{array}$ & $\mathbf{P}_{4}$ & $\begin{array}{l}\mathbf{L} \\
\mathbf{W}\end{array}$ & $\begin{array}{l}50 \\
50\end{array}$ & $\begin{array}{l}7.05 \\
3.78\end{array}$ & $\begin{array}{l}6.3-7.8 \\
3.2-4.5\end{array}$ & $\begin{array}{l}.37 \\
.31\end{array}$ & $\begin{array}{l}5.2 \\
8.2\end{array}$ \\
\hline $\mathbf{M}^{1}$ & $\begin{array}{l}\mathbf{L} \\
\mathbf{W}\end{array}$ & $\begin{array}{l}51 \\
51\end{array}$ & $\begin{array}{l}7.85 \\
7.22\end{array}$ & $\begin{array}{l}7.3-8.5 \\
6.4-8.2\end{array}$ & $\begin{array}{l}.26 \\
.34\end{array}$ & $\begin{array}{l}3.3 \\
4.7\end{array}$ & $\mathrm{M}_{1}$ & $\begin{array}{l}\mathrm{L} \\
\mathrm{W}\end{array}$ & $\begin{array}{l}50 \\
50\end{array}$ & $\begin{array}{l}8.29 \\
5.18\end{array}$ & $\begin{array}{l}7.4-8.9 \\
4.6-5.9\end{array}$ & $\begin{array}{l}.32 \\
.29\end{array}$ & $\begin{array}{l}3.8 \\
5.5\end{array}$ \\
\hline $\mathbf{M}^{2}$ & $\begin{array}{l}\mathbf{L} \\
\mathbf{W}\end{array}$ & $\begin{array}{l}51 \\
51\end{array}$ & $\begin{array}{l}7.07 \\
7.19\end{array}$ & $\begin{array}{l}6.6-7.8 \\
6.4-7.9\end{array}$ & $\begin{array}{l}.27 \\
.34\end{array}$ & $\begin{array}{l}3.9 \\
4.8\end{array}$ & $\mathbf{M}_{2}$ & $\begin{array}{l}\mathrm{L} \\
\mathrm{W}\end{array}$ & $\begin{array}{l}50 \\
50\end{array}$ & $\begin{array}{l}7.19 \\
5.58\end{array}$ & $\begin{array}{l}6.7-7.9 \\
5.1-6.3\end{array}$ & $\begin{array}{l}.27 \\
.24\end{array}$ & $\begin{array}{l}3.8 \\
4.3\end{array}$ \\
\hline $\mathbf{M}^{3}$ & $\begin{array}{l}\mathbf{L} \\
\mathbf{W}\end{array}$ & $\begin{array}{l}51 \\
51\end{array}$ & $\begin{array}{l}6.10 \\
6.00\end{array}$ & $\begin{array}{l}5.5-6.8 \\
5.6-6.8\end{array}$ & $\begin{array}{l}.32 \\
.30\end{array}$ & $\begin{array}{l}5.3 \\
5.0\end{array}$ & $\mathrm{M}_{3}$ & $\begin{array}{l}\mathrm{L} \\
\mathrm{W}\end{array}$ & $\begin{array}{l}50 \\
50\end{array}$ & $\begin{array}{l}7.64 \\
5.52\end{array}$ & $\begin{array}{l}6.8-8.8 \\
5.1-6.1\end{array}$ & $\begin{array}{l}.41 \\
.25\end{array}$ & $\begin{array}{l}5.3 \\
4.6\end{array}$ \\
\hline
\end{tabular}

out of 124 measurements were significantly different suggests that there is no biologically significant dimorphism in the dentition of Indriidae.

The only previous attempt to quantify sexual dimorphism in Indriidae was by SwINDLER (1976, p. 40, p. 200-201), who found that males of Propithecus verreauxi tend to have significantly larger molars than females of the same species. SwINDLER's 
Table 5. Dental measurements and summary statistics for specimens of Avahi laniger of known sex (Significant differences between males and females marked with asterisk.)

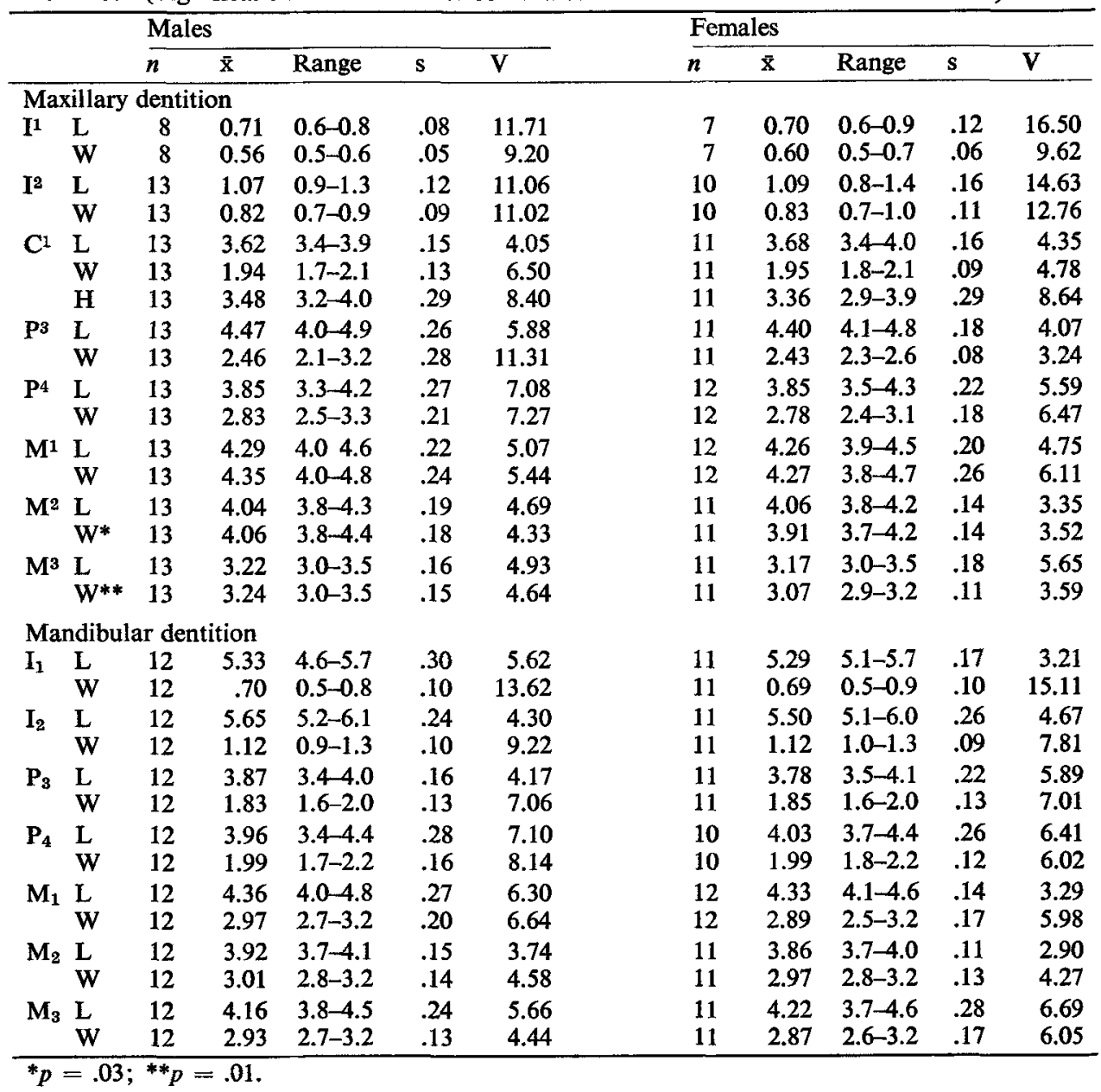

sample sizes were small ( $n=2-3$ for males, $n=5-7$ for females), and our larger samples of Propithecus verreauxi suggest that his conclusion regarding dimorphism is incorrect. As noted in the introduction, indriids are distinguished from many other primates by their lack of male dominance and limited intraspecific aggression, and this is probably the best explanation for the absence of sexual dimorphism in the dentition of Indriidae.

\section{Cranial Variation in INDRIIDAE}

Cranial measurements for all four species of Indriidae are given in Table 9. Interestingly, the length and the width of the cranium show surprisingly little variation. The coefficient of variation for cranial length ranges from a low of 3.3 in Indri to a high of 4.6 in Propithecus verreauxi. The coefficient of variation for cranial width ranges from a low of 4.1 in Indri to a high of 6.5 in P. verreauxi. In contrast, the 
Table 6. Dental measurements and summary statistics for specimens of Propithecus verreauxi of known sex (There are no significant differences between males and females.)

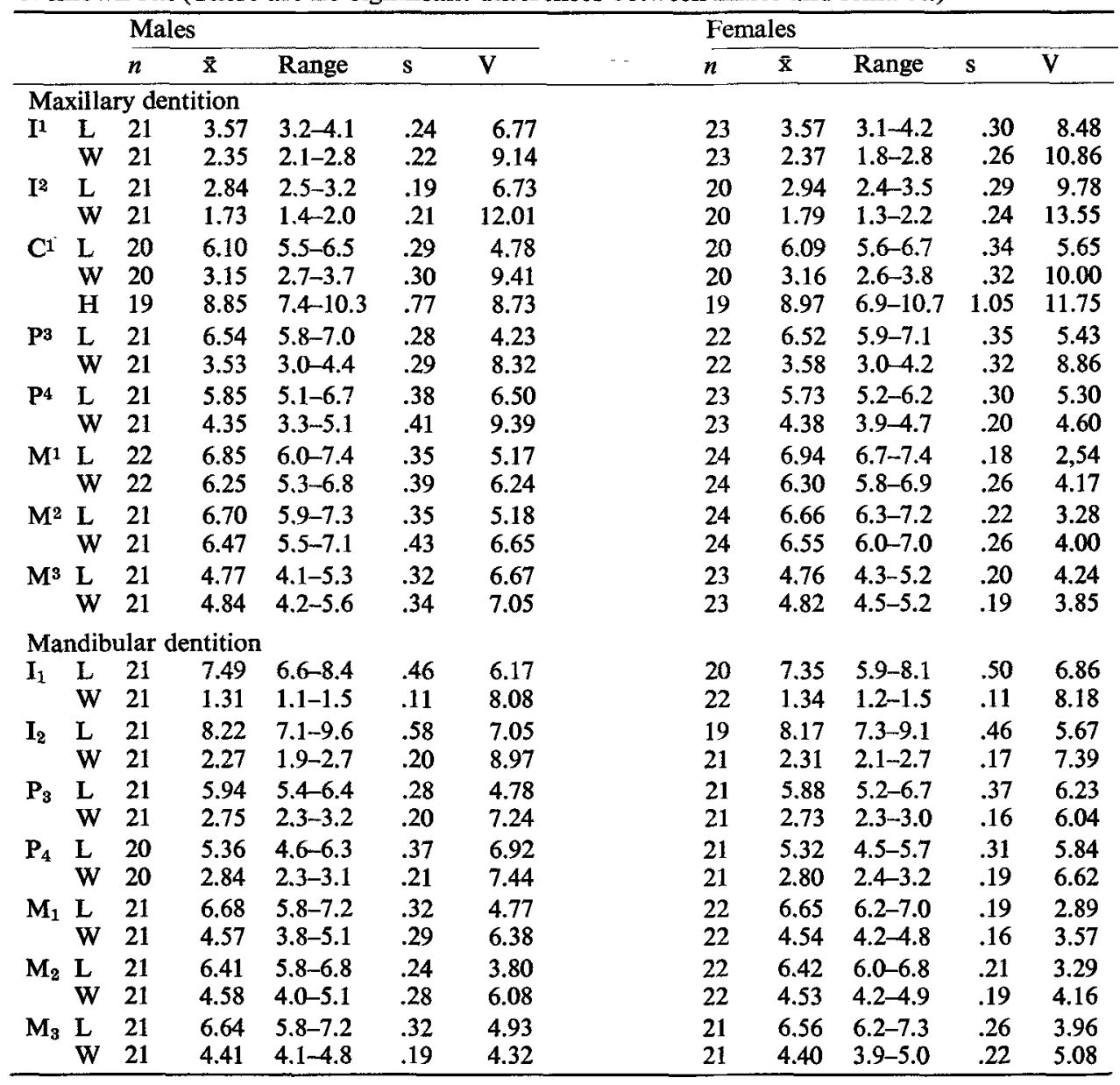

position of the temporal lines marking the separation of the edges of the temporal musculature is much more variable, with coefficients of variation ranging from 11.2 to 51.6.

Orbital diameter varies relatively little in Propithecus and Indri, but it is much more variable in Avahi. The width of the foramen magnum is consistently less variable than its height. Finally, interorbital breadth is moderately variable in all four species.

Statistics were calculated separately for specimens of known sex, and a summary of cranial measurements for male and female crania is given in Table 10. Male skulls are on the average slightly longer and wider than female skulls in Avahi and Propithecus, but the reverse is the case in Indri. However, the only statistically significant difference between male and female skulls is in the width of the foramen magnum in Indri. Since there is no consistent pattern of sexual dimorphism and there is only one significant difference out of a total of $36 t$-tests (which cannot be related to any significant functional difference between males and females), it seems unlikely that a 
Table 7. Dental measurements and summary statistics for specimens of Propithecus diadema of known sex (Significant differences between males and females marked with asterisk.)

\begin{tabular}{|c|c|c|c|c|c|c|c|c|c|c|c|}
\hline & \multicolumn{5}{|c|}{ Males } & \multicolumn{5}{|c|}{ Females } \\
\hline & & $n$ & $\overline{\mathrm{x}}$ & Range & $\mathrm{s}$ & $\mathrm{V}$ & $n$ & $\overline{\mathrm{x}}$ & Range & $\mathrm{s}$ & $\mathrm{V}$ \\
\hline \multicolumn{12}{|c|}{ Maxillary dentition } \\
\hline \multirow[t]{2}{*}{$1^{1}$} & L & 20 & 4.54 & $3.9-5.2$ & .35 & 7.68 & 14 & 4.71 & $4.1-5.0$ & .30 & 6.33 \\
\hline & W & 20 & 3,03 & $2.5-3.4$ & .24 & 7.80 & 14 & 3.04 & $2.6-3.3$ & .25 & 8.22 \\
\hline \multirow[t]{2}{*}{$\mathrm{I}^{2}$} & L & 20 & 3.17 & $2.6-3.6$ & .29 & 9.27 & 14 & 3.30 & $2.6-3.7$ & .31 & 9.51 \\
\hline & W & 20 & & $1.8-2.6$ & .24 & 10.95 & 14 & 2.04 & $1.4-2.3$ & .31 & 15.12 \\
\hline \multirow[t]{3}{*}{$\mathrm{C}^{1}$} & $\mathrm{~L}$ & 20 & 6.13 & $5.2-7.1$ & .49 & 7.98 & 12 & 6.00 & $5.3-6.6$ & .42 & 7.04 \\
\hline & $\mathrm{W}^{*}$ & 20 & 3.59 & $2.9-4.0$ & .31 & 8.60 & 12 & 3.35 & $3.1-3.7$ & .23 & 6.91 \\
\hline & $\mathbf{H}$ & 20 & 9.47 & $7.3-11.0$ & .95 & 10.02 & 12 & 10.20 & $7.2-11.8$ & 1.13 & 11.11 \\
\hline \multirow[t]{2}{*}{$\mathbf{P}^{3}$} & $\mathrm{~L}$ & 22 & 6.04 & $5.2-6.7$ & .34 & 5.71 & 13 & 6.22 & $5.4-6.9$ & .40 & 6.46 \\
\hline & W & 22 & 3.77 & $3.3-4.9$ & .39 & 10.47 & 13 & 3.88 & $3.4-4.4$ & .30 & 7.71 \\
\hline \multirow[t]{2}{*}{$\mathrm{P}^{4}$} & $\mathrm{~L}$ & 22 & 5.57 & $4.6-7.5$ & .64 & 11.42 & 14 & 5.69 & $5.2-6.3$ & .30 & 5.31 \\
\hline & W & 22 & 4.91 & $4.5-7.0$ & .51 & 10.29 & 14 & 4.78 & $4.1-5.2$ & .31 & 6.52 \\
\hline \multirow[t]{2}{*}{$\mathbf{M}^{1}$} & L & 22 & 7.21 & $6.5-7.9$ & .33 & 4.55 & 14 & 7.34 & $6.5-7.9$ & .37 & 4.98 \\
\hline & W & 22 & 6.97 & $4.0-7.9$ & .79 & 11.38 & 14 & 7.06 & $6.2-7.7$ & .40 & 5.71 \\
\hline \multirow[t]{2}{*}{$\mathbf{M}^{2}$} & $\mathrm{~L}$ & 21 & 7.19 & $6.4-7.9$ & .37 & 5.19 & 14 & 7.34 & $6.5-8.0$ & .43 & 5.82 \\
\hline & W & 21 & 7.35 & $6.5-8.3$ & .49 & 6.71 & 14 & 7.24 & $6.4-7.8$ & .38 & 5.21 \\
\hline \multirow[t]{2}{*}{$\mathbf{M}^{3}$} & L & 20 & 5.68 & $5.0-6.6$ & .33 & 5.83 & 13 & 5.65 & $4.9-6.2$ & .35 & 6.12 \\
\hline & W & 20 & 5.68 & $5.2-6.3$ & .30 & 5.27 & 13 & 5.57 & $5.1-6.1$ & .28 & 5.10 \\
\hline \multicolumn{12}{|c|}{ Mandibular dentition } \\
\hline \multirow[t]{2}{*}{$\mathrm{I}_{1}$} & L & 19 & 8.42 & $6.8-9.9$ & .86 & 10.17 & 12 & 8.83 & $8.1-9.6$ & .50 & 5.72 \\
\hline & W & 20 & 1.54 & $1.3-1.8$ & .19 & 12.36 & 12 & 1.65 & $1.4-1.9$ & .17 & 10.17 \\
\hline \multirow[t]{2}{*}{$I_{2}$} & $\mathrm{~L}$ & 20 & 9.29 & $8.0-10.8$ & .77 & 8.24 & 13 & 9.70 & $8.8-10.3$ & .39 & 3.97 \\
\hline & W & 21 & 2.80 & $2.2-3.3$ & .28 & 9.97 & 13 & 2.85 & $2.5-3.2$ & .23 & 7.94 \\
\hline \multirow[t]{2}{*}{$\mathbf{P}_{3}$} & L & 21 & 5.78 & $5.2-6.6$ & .35 & 6.01 & 12 & 6.02 & $5.2-6.4$ & .34 & 5.57 \\
\hline & W & 21 & 3.03 & $2.7-3.6$ & .26 & 8.55 & 12 & 3.19 & $2.8-3.5$ & .21 & 6.61 \\
\hline \multirow[t]{2}{*}{$\mathbf{P}_{4}$} & L & 21 & 4.92 & $4.4-5.4$ & .29 & 5.99 & 13 & 4.96 & $4.3-5.5$ & .38 & 4.80 \\
\hline & W & 21 & 3.16 & $2.5-3.7$ & .26 & 8.29 & 13 & 3.15 & $2.4-3.5$ & .30 & 9.48 \\
\hline \multirow[t]{2}{*}{$\mathbf{M}_{1}$} & L & 22 & 6.86 & $6.2-7.5$ & .33 & 4.88 & 13 & 6.95 & $6.1-7.5$ & .40 & 5.72 \\
\hline & W & 22 & 5.03 & $4.6-5.7$ & .26 & 5.11 & 13 & 5.01 & $4.4-5.4$ & .30 & 5.96 \\
\hline \multirow[t]{2}{*}{$\mathbf{M}_{2}$} & L & 22 & 7.05 & $6.5-7.7$ & .32 & 4.48 & 13 & 7.10 & $6.4-7.5$ & .30 & 4.23 \\
\hline & W & 22 & 5.24 & $4.8-5.8$ & .26 & 4.95 & 13 & 5.18 & $4.5-5.6$ & .29 & 5.67 \\
\hline \multirow[t]{2}{*}{$\mathbf{M}_{3}$} & L & 20 & 7.69 & $6.4-8.4$ & .46 & 6.02 & 12 & 7.68 & $6.8-8.2$ & .42 & 5.42 \\
\hline & W & 20 & 5.24 & $4.7-5.8$ & .29 & 5.48 & 12 & 5.13 & $4.5-5.5$ & .25 & 4.80 \\
\hline
\end{tabular}

sex difference the width of the foramen magnum in our sample of Indri is significant biologically. The data appear to demonstrate, on the other hand, that there is no significant sexual dimorphism in the crania of living Indriidae.

\section{Variation of Tooth Crown Area}

The final area investigated in this study was the pattern of variation in the crosssectional crown area of each tooth. Patterns of variation in tooth length and tooth width considered separately were presented above in Tables 1-4. Here we consider a pattern of covariation in tooth length and width.

In a previous study, Gingerich (1974) proposed that the log of the length multiplied by width of the least variable cheek tooth should be used to estimate specimen size in fossil mammals. This has an advantage over using length or width alone in that the estimate is based on two independent (but correlated) measurements of each tooth. Since the length and the width of $M_{1}$ tend to be less variable than the length 
Table 8. Dental measurements and summary statistics for specimens of Indri indri of known sex (Significant differences between males and females marked with asterisk.)

\begin{tabular}{|c|c|c|c|c|c|c|c|c|c|c|c|}
\hline & & \multicolumn{5}{|c|}{ Males } & \multicolumn{5}{|c|}{ Females } \\
\hline & & $n$ & $\overline{\mathbf{x}}$ & Range & s & $\mathrm{V}$ & $n$ & $\overline{\mathrm{x}}$ & Range & s & V \\
\hline \multicolumn{12}{|c|}{ Maxillary dentition } \\
\hline I & $\begin{array}{l}\mathbf{L} \\
\mathbf{W}\end{array}$ & $\begin{array}{l}16 \\
16\end{array}$ & $\begin{array}{l}4.16 \\
2.10\end{array}$ & $\begin{array}{l}3.3-5.0 \\
1.8-2.6\end{array}$ & $\begin{array}{l}.40 \\
.19\end{array}$ & $\begin{array}{l}9.58 \\
9.20\end{array}$ & $\begin{array}{l}11 \\
11\end{array}$ & $\begin{array}{l}4.25 \\
2.15\end{array}$ & $\begin{array}{l}3.7-4.7 \\
2.0-2.3\end{array}$ & $\begin{array}{l}.33 \\
.10\end{array}$ & $\begin{array}{l}7.82 \\
4.81\end{array}$ \\
\hline$I^{2}$ & $\begin{array}{l}\text { L } \\
\text { W }\end{array}$ & $\begin{array}{l}16 \\
16\end{array}$ & $\begin{array}{l}4.31 \\
2.23\end{array}$ & $\begin{array}{l}3.3-4.9 \\
1.9-2.7\end{array}$ & $\begin{array}{l}.39 \\
.21\end{array}$ & $\begin{array}{l}8.96 \\
9.36\end{array}$ & $\begin{array}{l}10 \\
10\end{array}$ & $\begin{array}{l}4.44 \\
2.27\end{array}$ & $\begin{array}{l}4.2-4.9 \\
1.9-2.5\end{array}$ & $\begin{array}{l}.24 \\
.19\end{array}$ & $\begin{array}{l}5.33 \\
8.22\end{array}$ \\
\hline \multirow[t]{3}{*}{$\mathrm{C}^{1}$} & $\mathbf{L}$ & 16 & 7.09 & $6.4-7.6$ & .36 & 5.10 & 11 & 6.95 & $6.4-7.8$ & .41 & 5.90 \\
\hline & W & 16 & 3.85 & $3.4-4.2$ & .25 & 6.43 & 11 & 3.88 & $3.4-4.1$ & .20 & 5.13 \\
\hline & H & 16 & 7.14 & $6.2-8.4$ & .57 & 7.96 & 11 & 7.19 & $5.9-8.3$ & .78 & 10.87 \\
\hline \multirow[t]{2}{*}{$\mathbf{P}^{3}$} & $\mathbf{L}$ & 16 & 7.61 & $7.0-8.7$ & .40 & 5.25 & 11 & 7.69 & $7.3-8.4$ & .32 & 4.17 \\
\hline & W & 16 & 4.38 & $3.8-4.9$ & .31 & 7.16 & 11 & 4.56 & 3.85 .0 & .42 & 9.10 \\
\hline \multirow[t]{2}{*}{$\mathbf{P}^{4}$} & $\mathrm{~L}$ & 16 & 6.98 & $6.6-8.0$ & .37 & 5.29 & 11 & 6.99 & $6.6-7.3$ & .21 & 3.03 \\
\hline & W & 16 & 5.66 & $5.0-6.4$ & .37 & 6.54 & 11 & 5.73 & $5.0-6.3$ & .37 & 6.54 \\
\hline \multirow[t]{2}{*}{$\mathbf{M}^{1}$} & $\mathbf{L}$ & 16 & 7.84 & $7.4-8.3$ & .31 & 3.92 & 11 & 7.92 & $7.6-8.2$ & .21 & 2.70 \\
\hline & W & 16 & 7.21 & $6.4-8.2$ & .38 & 5.28 & 11 & 7.39 & $6.6-8.1$ & .40 & 5.39 \\
\hline \multirow[t]{2}{*}{$\mathbf{M}^{2}$} & $\mathrm{~L}^{*}$ & 16 & 6.97 & $6.7-7.8$ & .29 & 4.22 & 11 & 7.25 & $6.9-7.7$ & .28 & 3.82 \\
\hline & W & 16 & 7.19 & $6.4-7.7$ & .35 & 4.87 & 11 & 7.35 & $6.8-7.9$ & .36 & 4.96 \\
\hline \multirow[t]{2}{*}{$M^{3}$} & $\mathrm{~L}^{* *}$ & 16 & 6.01 & $5.6-6.5$ & .23 & 3.88 & 11 & 6.25 & $5.8-6.8$ & .27 & 4.32 \\
\hline & W & 16 & 5.98 & $5.6-6.7$ & .30 & 4.98 & 11 & 6.10 & $5.7-6.5$ & .24 & 3.88 \\
\hline \multicolumn{12}{|c|}{ Mandibular dentition } \\
\hline \multirow[t]{2}{*}{$\mathbf{I}_{1}$} & $\mathrm{~L}$ & 15 & 10.69 & $9.8-11.4$ & .53 & 4.94 & 10 & 10.73 & $9.0-11.8$ & .92 & 8.54 \\
\hline & W & 15 & 1.56 & $1.3-1.7$ & .13 & 8.32 & 11 & 1.57 & $1.3-1.8$ & .14 & 9.03 \\
\hline \multirow[t]{2}{*}{$\mathbf{I}_{2}$} & L & 15 & 11.24 & $10.2-12.1$ & .49 & 4.37 & 10 & 11.28 & $10.1-12.2$ & .65 & 5.79 \\
\hline & W & 15 & 2.61 & $2.2-2.9$ & .18 & 6.92 & 11 & 2.65 & $2.4-2.9$ & .18 & 6.63 \\
\hline \multirow[t]{2}{*}{$\mathbf{P}_{3}$} & $\mathrm{~L}$ & 16 & 6.94 & $5.9-7.6$ & .43 & 6.18 & 11 & 7.08 & $6.3-7.7$ & .39 & 5.50 \\
\hline & W & 16 & & & .26 & 7.57 & 11 & & & .16 & $\begin{array}{l}4.47 \\
554\end{array}$ \\
\hline $\mathbf{P}_{4}$ & $\begin{array}{l}\text { L } \\
\text { W }\end{array}$ & $\begin{array}{l}16 \\
16\end{array}$ & $\begin{array}{l}7.03 \\
3.62\end{array}$ & $\begin{array}{l}6.4-7.5 \\
3.2-4.5\end{array}$ & $\begin{array}{l}.29 \\
.33\end{array}$ & $\begin{array}{l}4.08 \\
9.15\end{array}$ & $\begin{array}{l}11 \\
11\end{array}$ & $\begin{array}{l}6.86 \\
3.77\end{array}$ & $\begin{array}{l}6.3-7.4 \\
3.4-4.2\end{array}$ & $\begin{array}{l}.38 \\
.23\end{array}$ & $\begin{array}{l}5.54 \\
6.17\end{array}$ \\
\hline \multirow[t]{2}{*}{$\mathbf{M}_{1}$} & $\mathbf{L}$ & 16 & 8.24 & $7.7-8.8$ & .31 & 3.78 & 11 & 8.37 & $7.9-8.8$ & .30 & 3.55 \\
\hline & W & 16 & 5.15 & $4.6-5.7$ & .33 & 6.50 & 11 & 5.18 & $4.9-5.7$ & .24 & 4.63 \\
\hline \multirow{2}{*}{$\mathbf{M}_{2}$} & $\mathbf{L}$ & 16 & 7.17 & $6.8-7.9$ & .31 & 4.31 & 11 & 7.37 & $7.1-7.7$ & .20 & 2.72 \\
\hline & W & 16 & 5.53 & $5.1-6.0$ & .23 & 4.39 & 11 & 5.58 & $5.3-6.0$ & .23 & 4.07 \\
\hline \multirow{2}{*}{$\mathbf{M}_{3}$} & $\mathbf{L}$ & 16 & 7.53 & $6.8-8.4$ & .40 & 5.30 & 11 & 7.73 & $7.3-8.5$ & .32 & 4.39 \\
\hline & W & 16 & 5.49 & $5.2-6.0$ & .25 & 4.60 & 11 & 5.58 & $5.3-6.0$ & .24 & 4.23 \\
\hline
\end{tabular}

and width of other cheek teeth in primates and other mammals, it was inferred that the product of length and width for $M_{1}$ would also be less variable than this product for other cheek teeth, but this was never tested. In this study of Indriidae, the length and width of $\mathrm{M}_{2}^{2}$ have been found to be the least variable of the cheek teeth, and we can test whether or not the product of length multiplied by width is also the least variable for $\mathrm{M}_{2}^{2}$.

It is customary to use logarithms of tooth size when comparing species to make the variability of large species comparable to that of small species. Hence, the log of the product of length multiplied by width is the quantity summarized in Table 11. The standard deviation of the $\log$ of crown area multiplied by 100 is equivalent to the coefficient of variation of crown area (LEWONTIN, 1966), and the standard deviations listed in Table 11 can thus be converted to coefficients of variation of the product of length multiplied by width for each tooth. As expected, the product of length multiplied by width is generally the least variable for $M_{2}^{2}$ among the cheek teeth. As was 
Table 9. Cranial measurements and summary statistics for all species of Indriidae, with male female, and specimens of unknown sex combined

\begin{tabular}{|c|c|c|c|c|c|}
\hline & $n$ & $\overline{\mathbf{x}}$ & Range & $\mathrm{s}$ & $\mathbf{V}$ \\
\hline \multicolumn{6}{|l|}{ Avahi laniger } \\
\hline Skull length & 30 & 47.87 & $43.0-50.9$ & 1.91 & 4.0 \\
\hline Skull width & 28 & 39.21 & $35.2-42.5$ & 1.76 & 4.5 \\
\hline Interorbital breadth & 29 & 11.27 & $9.8-14.4$ & 1.07 & 9.5 \\
\hline Anterior temporal separation & 27 & 16.56 & $12.4-19.9$ & 1.85 & 11.2 \\
\hline Posterior temporal separation & 26 & 9.70 & $3.6-15.0$ & 2.88 & 29.7 \\
\hline Orbital height & 30 & 16.29 & $7.6-18.1$ & 2.39 & 14.7 \\
\hline Orbital width & 30 & 15.76 & $7.0-17.3$ & 2.42 & 15.4 \\
\hline Foramen magnum width & 20 & 8.48 & $7.9-9.2$ & .32 & 3.8 \\
\hline Foramen magnum height & 20 & 8.73 & $7.8-9.7$ & .58 & 6.6 \\
\hline \multicolumn{6}{|l|}{ Propithecus verreauxi } \\
\hline Skull length & 51 & 75.04 & $67.0-88.0$ & 3.48 & 4.6 \\
\hline Skull width & 54 & 54.63 & $45.4-61.4$ & 3.54 & 6.5 \\
\hline Interorbital breadth & 53 & 20.22 & $14.7-27.2$ & 2.60 & 12.8 \\
\hline Anterior temporal separation & 50 & 18.04 & $7.9-26.2$ & 3.56 & 19.7 \\
\hline Posterior temporal separation & 46 & 10.08 & $0.0-19.3$ & 5.20 & 51.6 \\
\hline Orbital height & 54 & 20.26 & $17.0-22.8$ & 1.36 & 6.7 \\
\hline Orbital width & 53 & 18.83 & $16.2-20.0$ & .90 & 4.8 \\
\hline Foramen magnum width & 34 & 12.22 & $11.0-13.8$ & .74 & 6.1 \\
\hline Foramen magnum height & 34 & 11.58 & $8.7-13.8$ & .92 & 8.0 \\
\hline \multicolumn{6}{|l|}{ Propithecus diadema } \\
\hline Skull length & 34 & 82.32 & $77.0-92.0$ & 3.46 & 4.2 \\
\hline Skull width & 38 & 57.81 & $50.0-62.8$ & 3.06 & 5.3 \\
\hline Interorbital breadth & 38 & 19.55 & $16.4-23.1$ & 1.60 & 8.2 \\
\hline Anterior temporal separation & 36 & 24.37 & $18.6-30.0$ & 3.06 & 12.6 \\
\hline Posterior temporal separation & 31 & 20.29 & $14.4-27.9$ & 3.34 & 16.5 \\
\hline Orbital height & 39 & 21.93 & $19.9-23.5$ & 1.02 & 4.7 \\
\hline Orbital width & 39 & 20.92 & $18.9-22.5$ & .97 & 4.6 \\
\hline Foramen magnum width & 26 & 13.90 & $12.5-15.1$ & .77 & 5.6 \\
\hline Foramen magnum height & 26 & 13.08 & $9.7-14.8$ & 1.03 & 7.9 \\
\hline \multicolumn{6}{|l|}{ Indri indri } \\
\hline Skull length & 49 & 95.51 & $89.0-102.0$ & 3.18 & 3.3 \\
\hline Skull width & 49 & 61.61 & $53.6-65.8$ & 2.53 & 4.1 \\
\hline Interorbital breadth & 49 & 19.55 & $8.7-24.4$ & 2.76 & 14.1 \\
\hline Anterior temporal separation & 49 & 26.41 & $13.8-35.4$ & 4.61 & 17.5 \\
\hline Posterior temporal separation & 46 & 14.71 & $3.3-24.8$ & 5.32 & 36.1 \\
\hline Orbital height & 51 & 23.39 & $21.4-24.8$ & .85 & 3.7 \\
\hline Orbital width & 51 & 22.75 & $20.2-24.5$ & .89 & 3.9 \\
\hline Foramen magnum width & 27 & 13.43 & $12.2-15.0$ & .82 & 6.1 \\
\hline Foramen magnum height & 27 & 11.97 & $9.6-13.8$ & 1.14 & 9.5 \\
\hline
\end{tabular}

found above when length and width were considered separately, the upper canine and $P_{3}$ also tend to be less variable than the neighboring teeth. This is discussed at greater length in the following section on dental fields.

\section{DENTAL FIELDS-A DISCUSSION}

Tables 1-11 summarize the main features of dental and cranial variation in the Indriidae. One particular paftern in this variation deserves special emphasis. BUTLER (1939) was the first to point out a correspondence between the mode of differentiation of teeth along the mammalian tooth row and the determination and localization of development in other organ systems. He borrowed the term "morphogenetic field" 


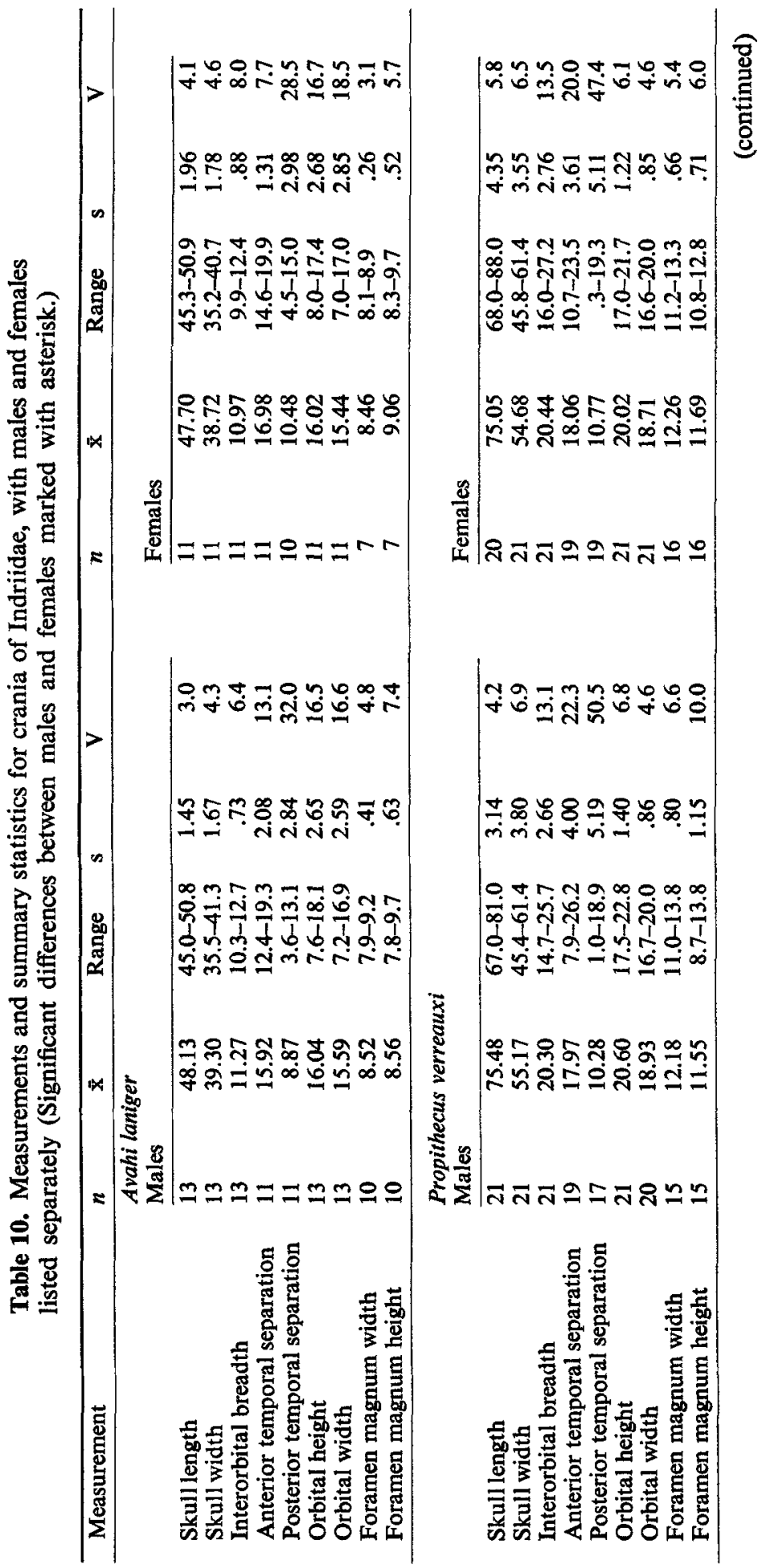




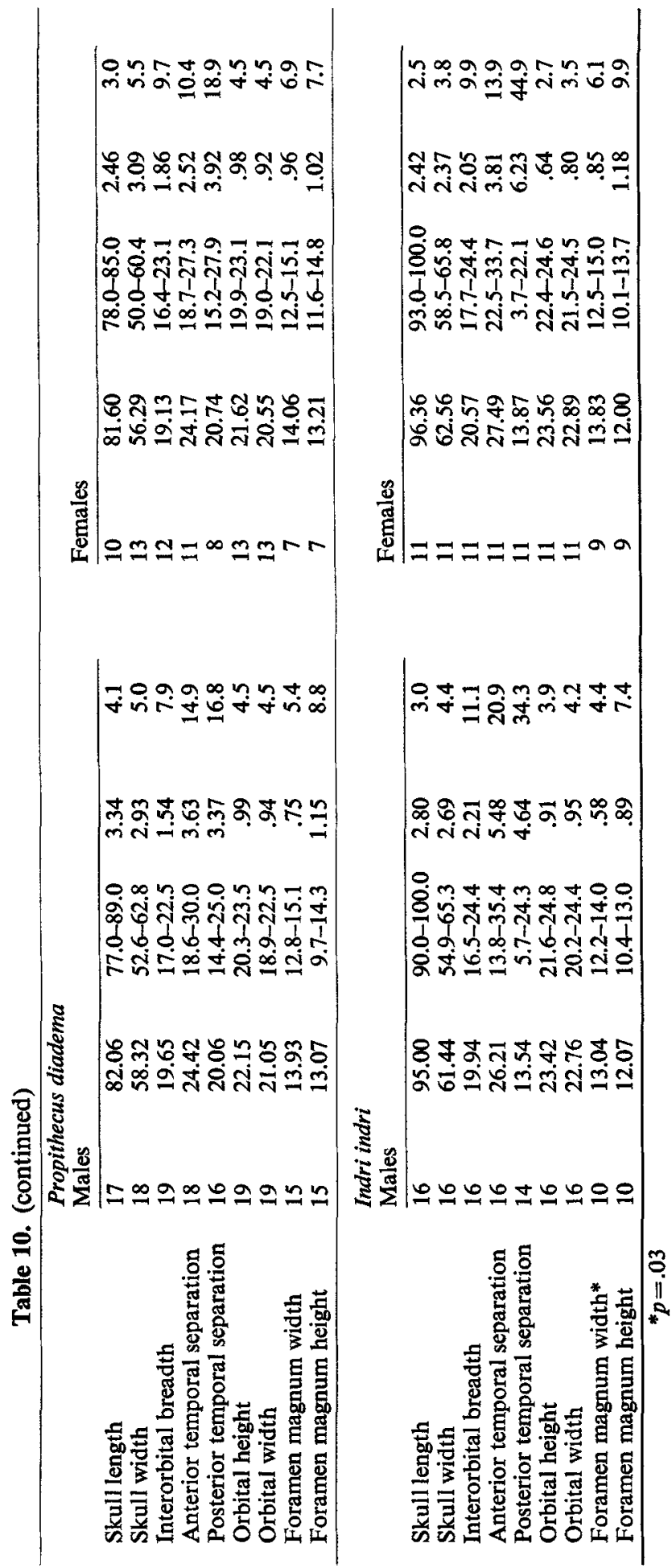


Table 11. Summary statistics for log crown area of teeth of Indriidae

\begin{tabular}{|c|c|c|c|c|c|c|c|c|c|}
\hline & \multicolumn{4}{|c|}{ Maxillary dentition } & & \multicolumn{4}{|c|}{ Mandibular dentition } \\
\hline & $n$ & $\bar{x}$ & Range & $\mathbf{s}$ & & $\bar{n}$ & $\overline{\mathbf{x}}$ & Range & $\mathbf{s}$ \\
\hline \multicolumn{10}{|c|}{ Avahi laniger } \\
\hline$I^{1}$ & 18 & -0.94 & $-1.6--0.6$ & .23 & $I_{1}$ & 30 & 1.29 & $1.0-1.6$ & .16 \\
\hline $\mathbf{I}^{2}$ & 28 & -0.16 & $-0.7-0.2$ & .23 & $\mathbf{I}_{2}$ & 30 & 1.81 & $1.5-2.0$ & .10 \\
\hline $\mathrm{C}$ & 30 & 1.95 & $1.7-2.1$ & .09 & & & & & \\
\hline $\mathbf{P}^{3}$ & 30 & 2.35 & $2.1-2.8$ & .12 & $\mathbf{P}_{\mathbf{3}}$ & 30 & 1.94 & $1.8-2.1$ & .10 \\
\hline $\mathbf{P}^{4}$ & 31 & 2.36 & $2.1-2.6$ & .12 & $\mathbf{P}_{4}$ & 29 & 2.05 & $1.8-2.2$ & .12 \\
\hline $\mathbf{M}^{1}$ & 31 & 2.90 & $2.7-3.1$ & .10 & $\mathbf{M}_{1}$ & 31 & 2.54 & $2.4-2.7$ & .10 \\
\hline $\mathbf{M}^{2}$ & 30 & 2.78 & $2.6-2.9$ & .07 & $\mathbf{M}_{2}$ & 30 & 2.45 & $2.3-2.6$ & .07 \\
\hline $\mathbf{M}^{3}$ & 30 & 2.30 & $2.1-2.4$ & .10 & $\mathbf{M}_{3}$ & 30 & 2.49 & $2.3-2.7$ & .10 \\
\hline \multicolumn{10}{|c|}{ Propithecus verreauxi } \\
\hline$I^{1}$ & 55 & 2.13 & $1.8-2.5$ & .15 & $\mathbf{I}_{\mathbf{1}}$ & 50 & 2.29 & $2.0-2.5$ & .12 \\
\hline$I^{2}$ & 54 & 1.61 & $1.3-2.0$ & .18 & $I_{2}$ & 50 & 2.93 & $2.7-3.2$ & .12 \\
\hline C & 53 & 2.95 & $2.7-3.2$ & .12 & & & & & \\
\hline P3 & 56 & 3.13 & $2.9-3.4$ & .11 & $\mathbf{P}_{\mathbf{3}}$ & 55 & 2.77 & $2.5-3.0$ & .09 \\
\hline $\mathbf{P}^{4}$ & 58 & 3.22 & $2.8-3.5$ & .12 & $\mathbf{P}_{4}$ & 54 & 2.70 & $2.4-2.9$ & .11 \\
\hline $\mathbf{M}^{1}$ & 60 & 3.77 & $3.5-3.9$ & .08 & $\mathbf{M}_{1}$ & 56 & 3.41 & $3.1-3.6$ & .08 \\
\hline $\mathbf{M}^{2}$ & 59 & 3.76 & $3.2-3.9$ & .11 & $\mathbf{M}_{2}$ & 56 & 3.37 & $3.1-3.5$ & .07 \\
\hline $\mathbf{M}^{3}$ & 56 & 3.13 & $2.8-3.4$ & .10 & $\mathbf{M}_{3}$ & 55 & 3.36 & $3.2-3.6$ & .08 \\
\hline \multicolumn{10}{|c|}{ Propithecus diadema } \\
\hline $\mathrm{I}^{1}$ & 42 & 2.63 & $2.4-2.9$ & .14 & $I_{1}$ & 37 & 2.60 & $2.2-2.9$ & .17 \\
\hline $\mathbf{I}^{2}$ & 42 & 1.90 & $1.4-2.2$ & .20 & $\mathbf{I}_{2}$ & 40 & 3.28 & $2.9-3.5$ & .14 \\
\hline C & 39 & 3.05 & $2.8-3.3$ & .12 & & & & & \\
\hline $\mathbf{P}^{3}$ & 43 & 3.15 & $2.9-3.3$ & .12 & $\mathbf{P}_{\mathbf{3}}$ & 40 & 2.89 & $2.6-3.1$ & .13 \\
\hline $\mathbf{P}^{4}$ & 44 & 3.30 & $3.1-4.0$ & .15 & $\mathbf{P}_{4}$ & 41 & 2.74 & $2.3-2.9$ & .13 \\
\hline $\mathrm{M}^{1}$ & 44 & 3.92 & $3.4-4.1$ & .14 & $\mathbf{M}_{1}$ & 42 & 3.54 & $3.3-3.8$ & .10 \\
\hline $\mathbf{M}^{2}$ & 43 & 3.97 & $3.7-4.2$ & .10 & $\mathbf{M}_{2}$ & 42 & 3.61 & $3.4-3.8$ & .09 \\
\hline $\mathbf{M}^{3}$ & 41 & 3.46 & $3.2-3.7$ & .11 & $\mathbf{M}_{3}$ & 39 & 3.69 & $3.4-4.0$ & .11 \\
\hline \multicolumn{10}{|c|}{ Indri indri } \\
\hline $\mathbf{I}^{1}$ & 46 & 2.16 & $1.8-2.5$ & .16 & $\mathbf{I}_{1}$ & 48 & 2.84 & $2.5-3.1$ & .13 \\
\hline $\mathbf{I}^{2}$ & 47 & 2.29 & $1.9-2.6$ & .15 & $\mathbf{I}_{2}$ & 48 & 3.41 & $3.2-3.6$ & .10 \\
\hline C & 51 & 3.28 & $3.1-3.5$ & .10 & & & & & \\
\hline $\mathbf{P}^{3}$ & 51 & 3.50 & $3.3-3.8$ & .12 & $\mathbf{P}_{3}$ & 50 & 3.21 & $2.9-3.4$ & .09 \\
\hline $\mathrm{P}^{4}$ & 51 & 3.65 & $3.4-3.9$ & .10 & $\mathbf{P}_{4}$ & 50 & 3.28 & $3.1-3.5$ & .11 \\
\hline M1 & 51 & 4.04 & $3.9-4.2$ & .07 & $\mathrm{M}_{1}$ & 50 & 3.76 & $3.5-4.0$ & .08 \\
\hline $\mathbf{M}^{2}$ & 51 & 3.93 & $3.8-4.1$ & .07 & $\mathbf{M}_{2}$ & 50 & 3.69 & $3.6-3.9$ & .07 \\
\hline $\mathbf{M}^{3}$ & 51 & 3.60 & $3.4-3.8$ & .09 & $\mathbf{M}_{3}$ & 50 & 3.74 & $3.6-4.0$ & .09 \\
\hline
\end{tabular}

Crown area expressed as $\log _{\mathrm{e}}(\mathrm{L} \times \mathrm{W})$. Standard deviation of $\log$ measurement(s) multiplied by 100 is equivalent to the coefficient of variation (V) of the original measurement (LewONTIN, 1966).

from embryology, applying it to the final form of the dentition. BUTLER $(1939$, p. 3) identified three subfields in the generalized mammalian dentition, which he identified as an "incisivization" field, a "caninization" field, and a "molarization" field. This field concept has been widely applied in studying tooth form (e.g., DAHLBERG, 1945); ontogenetic growth (VAN VALEN, 1962; MARshall \& BUTLeR, 1966), heritability (Alvesalo \& TigerstedT, 1974), and phylogeny (PATTERSon, 1949). In a recent paper on Indriidae, GINGERICH (1977) emphasized the functional aspect of fields in the dentition of mammals, with distinct incisor, canine, and cheek tooth fields functioning, respectively, in food acquisition, in display (and/or food acquisition), and in mastication. The data presented above clearly indicate the presence of two functional fields in the dentition of indriids, and it is possible that a third may be present as well. 


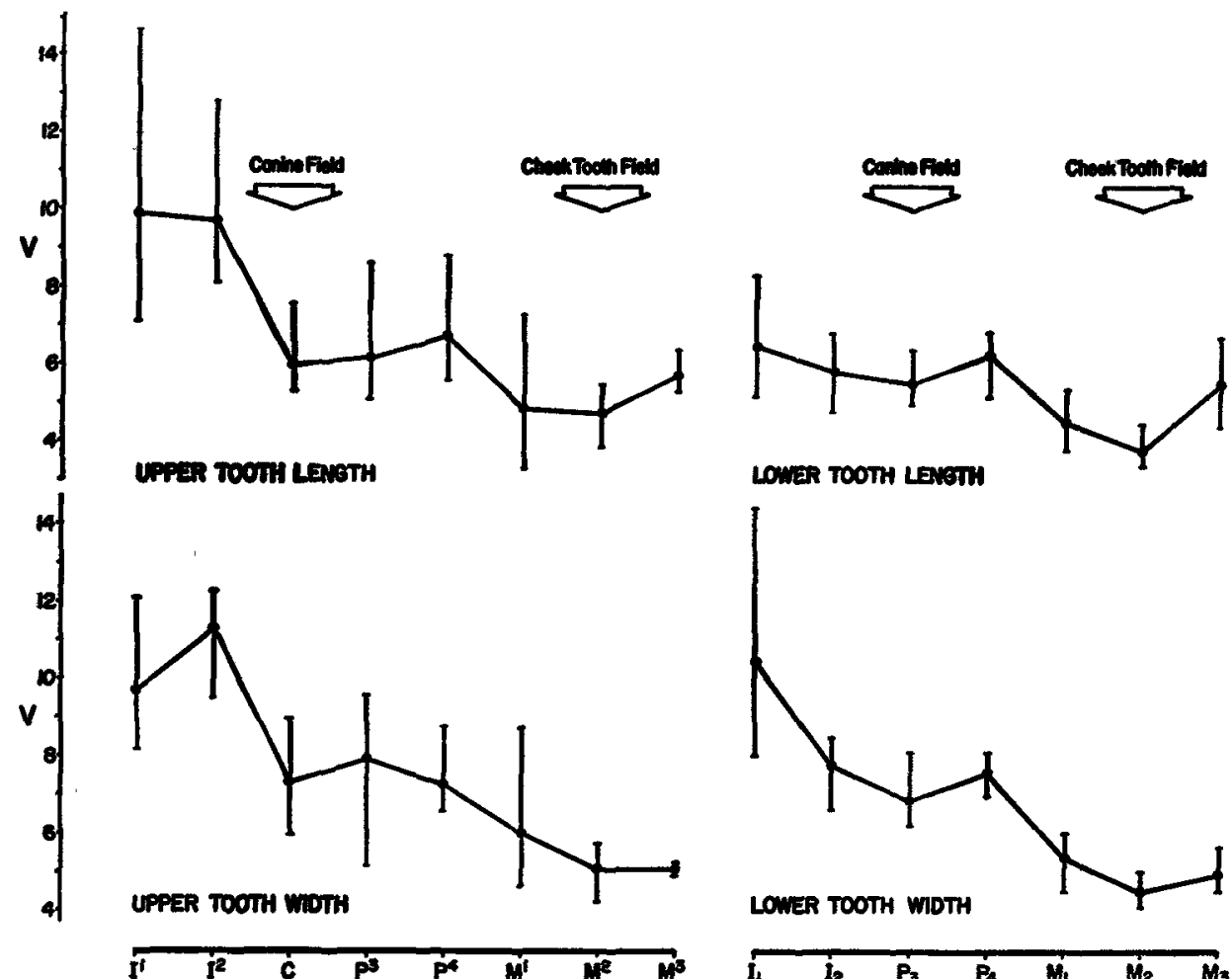

Fig. 2. Pattern of variability in upper and lower tooth length and width of Indriidae. $V$ is coefficient of variation from Tables 1-4. Solid circle is mean and vertical bar is range for each tooth position in four indriid species studied. Note low variability of upper canine and lower $\mathbf{P}_{3}$ (canine field), and low variability of $\mathbf{M}_{2}^{2}$ (center of cheek tooth field).

A consistent pattern is present when the coefficients of variation in tooth length and width are examined for each tooth position. This pattern is illustrated in Figure 2. Both upper and lower incisors have relatively large coefficients of variation. The upper canine and the caniniform lower premolar $\left(\mathrm{P}_{3}\right)$ have relatively small coefficients of variation. The fourth premolars $\left(\mathbf{P}_{4}^{4}\right)$ and the first and last molars $\left(\mathbf{M}_{1}^{1}\right.$ and $\left.\mathbf{M}_{3}^{3}\right)$ are relatively variable. The second molars $\left(\mathrm{M}_{2}^{2}\right)$, like the upper canine and lower $P_{3}$, have relatively small coefficients of variation.

Two fields of variation are apparent in Figure 2. The first is centered on the upper canine and occluding caniniform lower premolar, and corresponds to BUTLER's caninization field. Arrows in Figure 2 identify this canine field. The second field is centered on the second molars, and corresponds to BUTLER's molarization field. Arrows in Figure 2 identify this cheek tooth field.

The teeth in each field have a distinctive morphology in addition to their characteristic pattern of variability. The caniniform canine and premolar teeth in indriids have a simple pointed projecting crown, and occlude in a honing mechanism maintaining a sharp posterior edge on the upper canine. This canine morphology is found in many other primates, where it is usually exaggerated in males and serves a display function. Considering the absence of sexual dimorphism and the low level of male dominance 


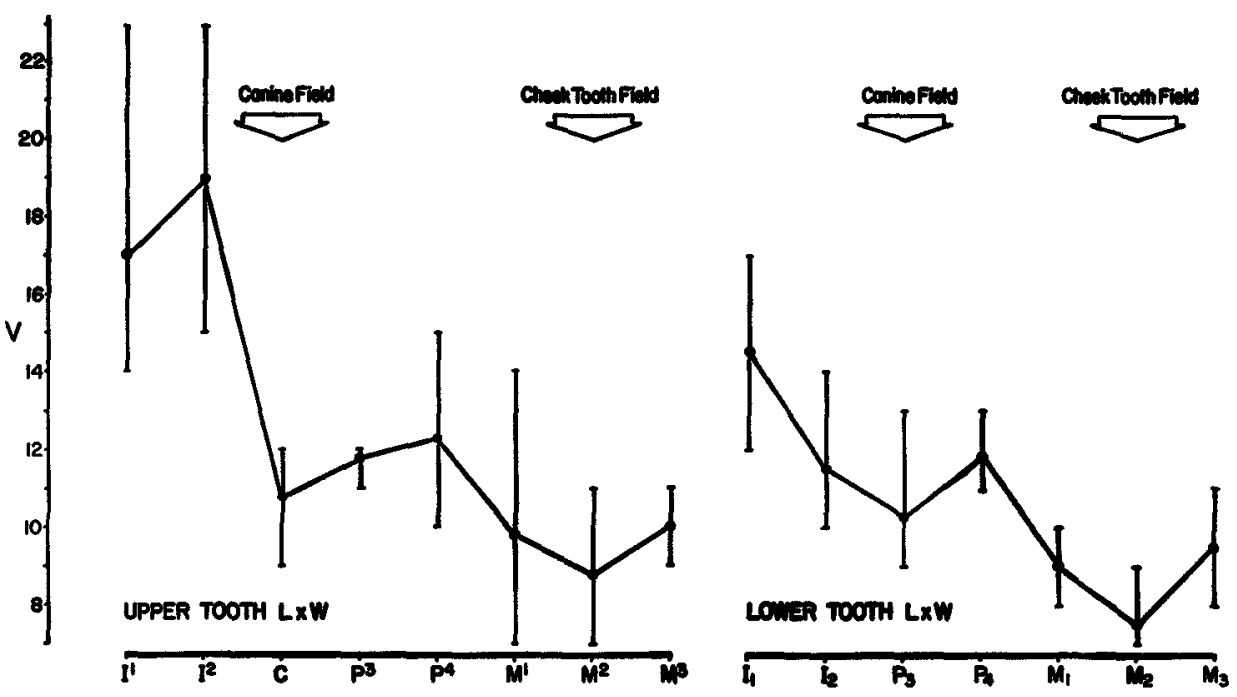

Fig. 3. Pattern of variability in upper and lower tooth crown area in Indriidae. $V$ is coefficient of variation calculated from Table 11. Solid circle is mean and vertical bar is range for each tooth position in four indriid species studied. Note canine and cheek tooth fields of low variability as in Figure 2.

and aggression in indriids, it is more likely that the canine field corresponds to an ingestive function during feeding by this family (as it may in other primates as well). Substantiation of this hypothesis will require very detailed observations on how indriids use their teeth during ingestion.

The cheek teeth of indriids have very pronounced shearing crests, with some development of planar grinding areas as well. They form a single integrated series in both the upper and lower jaws, with the pattern of molarization conforming very closely to the pattern of variability in length and width shown in Figure 2. This cheek tooth field functions almost exclusively in mastication.

The pattern of variability in crown area is plotted in Figure 3, and again the upper canine/lower $P_{3}$ and the second molars are centers of reduced variability. This pattern further supports the existence of two distinctive canine and cheek tooth fields in the indriid dentition.

The upper incisors in indriids (especially in Indri and $A v a h i$ ) tend to be somewhat reduced by comparison with anthropoids, and the lower incisors are modified into a dental scraper or tooth comb. In view of the integrated nature of the left and right incisors into a single functional field, it seems anomalous that the central incisors tend to be more variable than the lateral incisors. This is possibly a reflection of the absence of precise occlusion and the reduced functional importance of incisors by comparison with the canines and cheek teeth.

In an earlier study, GINGERICH (1974) showed that the first molars tend to be the least variable cheek teeth in a sample of 19 species of mammals, 15 of which were anthropoid primates. One can ask why the cheek tooth field is centered on the second molars in Indriidae instead of on the first molars as it appears to be in most primates 
and other mammals? The answer to this question is probably related to the fact that indriids have a reduced number of premolars and relatively short cheek tooth row. Shortening a symmetrical field from the front necessarily moves the center of the field backwards, and this appears to be what has happened in indriids.

GiNGERICH (1974) explained the tendency for the first molars to show the least variability in mammals as a result of two factors: (1) eruption sequence-the first molars erupt first, and thus are least affected by developing hormone differences between males and females and (2) position in tooth row-the central teeth in a functional field are the best integrated morphologically and thus the least variable. While the first explanation may be a factor in highly dimorphic species, it cannot be a factor in non-dimorphic Indriidae. The position effect appears to be of more general importance than the order of development and eruption in explaining the observed patterns of variation. Teeth in the center of a functional field are a more critical component of an animal's adaptation than teeth at the periphery and hence they are probably under more intense natural selection and genetic control, which is reflected by their reduced variability.

Finally, as a practical corollary of this discussion, an earlier conclusion that the first molar is the best tooth on which to base diagnoses of closely related fossil species (GINGERICH, 1974) requires some modification and generalization. Different groups of mammals have cheek tooth rows of differing lengths, with different functional adaptations. Dental variability in living representatives of any group should be studied, whenever possible, before attempting to diagnose closely related sympatric species of the group in the fossil record. In general, the first molars are probably the least variable and the best single teeth to use in studying size distributions in the fossil record, but in other groups like the Indriidae the second molars are least variable and thus the best for this purpose.

\section{SUMMARY}

Teeth and crania were measured in the four species of living Indriidae. This is the first study of dental and cranial variation in lemuroid prosimians to be based on large samples. The range, mean, and standard deviation of all measurements are tabulated, and this data is analyzed to determine the presence or absence of sexual dimorphism, and patterns of dental variability. Indriids show no consistent pattern of sexual dimorphism in dental or cranial measurements, and it is concluded that none of the four species is significantly dimorphic in size or in dental and cranial morphology. This absence of sexual dimorphism is presumably correlated with the relatively limited aggression and lack of male dominance in Indriidae.

Two distinct fields can be recognized in the patterns of dental variability in the indriid dentition. One field of low variability is centered on the upper canine and occluding lower anterior premolar. A second field of low variability is centered on the upper and lower second molars. These fields of low variability correspond to two functional fields in the dentition, a canine field and a cheek tooth field, and the low variability is plausibly explained as a result of more intense natural section and genetic control of dental elements most critical to an animal's feeding adaptation. 
Acknowledgments. We thank Drs. P. NAPIER, British Museum (Natural History) in London; F. K. JOUfFroy and J. LessertisseuR, Laboratoire d'Anatomie Comparée in Paris; L. B. Holthuis, Rijksmuseum van Natuurlijke Historie in Leiden; and P. HelwIG, Cleveland Museum of Natural History in Cleveland for access to specimens on which this study is based. Travel funds were provided by a Scott Turner Award in Earth Sciences through the Department of Geology and Mineralogy, The University of Michigan. We thank Mrs. GLADYS Newton for typing the manuscript and KaREN PAYNE for drawing Figure 1.

\section{REFERENCES}

Alvesalo, L. \& P. M. A. Tigerstedt, 1974. Heritabilities of human tooth dimensions. Hereditas, 77: 311-318.

Bauchot, R. \& H. Stephan, 1966. Donées nouvelles sur l'encephalization des insectivores et des prosimians. Mammalia, 30: 160-196.

BiggerstafF, R. H., 1966. Metric and taxonomic variations in the dentitions of two Asian cercopithecoid species: Macaca mulatta and Macaca speciosa. Am. J. Phys. Anthrop., 24: 231-138.

Butler, P. M., 1939. Studies of the mammalian dentition-differentiation of the post-canine dentition. Proc. Zool. Soc. Lond., 109: 1-36.

Dahlberg, A. A., 1945. The changing dentition of man. J. Am. Dent. Assoc., 32: 676-690.

Frisch, J. E., 1963. Sex differences in the canines of the gibbon (Hylobates lar). Primates, 4: 1-10.

GINGERICH, P. D., 1974. Size variability of the teeth in living mammals and the diagnosis of closely related sympatric fossil species. J. Paleont., 48: 895-903.

- 1977. Homologies of the anterior teeth in Indriidae and a functional basis for dental reduction in primates. Am. J. Phys. Anthrop., in press.

Godfrey, L., 1976. Dental reduction in the Indriidae. In: The Measures of Man, E. GILEs \& J. S. Friedlaender (eds.), Peabody Museum Press, Cambridge, pp. 109-139.

Jentink, F. A., 1887. Catalogue ostélogique des mammifères. Mus. d'Hist. Naturelle des PayBas, 9: 51-70.

Johanson, D. C., 1974. Some metric aspects of the permanent and deciduous dentition of the pygmy chimpanzee (Pan paniscus). Am. J. Phys. Anthrop., 41: 39-48.

LEUTENEGGER, W., 1971. Metric variability of the post-canine dentition in colobus monkeys. Am. J. Phys. Anthrop., 35: 91-100.

- 1973. Sexual dimorphism in the pelves of African lorises. Am. J. Phys. Anthrop., 38: $251-254$.

\& J. T. KeLley, 1977. Relationship of sexual dimorphism in canine size and body size to social, behavioral, and ecological correlates in anthropoid primates. Primates, 18: $117-136$.

Lewontin, R. C., 1966. On the measurement of relative variability. Syst. Zool., 15: 141-142.

Marshall, P. M. \& P. M. ButLer, 1966. Molar cusp development in the bat, Hipposideros beatus, with reference to the ontogenetic basis of occlusion. Arch. Oral. Biol., 11: 949965.

NAPIER, J. R. \& P. H. NAPIER, 1967. A Handbook of Living Primates. Academic Press, London.

Patterson, B., 1949. Rates of evolution in Taeniodonts. In: Genetics, Paleontology and Evolution, G. L. Jepsen, G. G. Simpson, \& E. Mayr (eds.), Princeton Univ. Press, Princeton, pp. 243-278.

PetTer, J. J., 1962. Recherches sur l'écologie et l'éthologie des Lémuriens malgaches. Mem. du Mus. Nat. de l'Hist. Naturelle Ser. A, 27: 1-146.

Prlbeam, D. R., 1969. Tertiary Pongidae of East Africa: evolutionary relationships and taxonomy. Peabody Mus. Nat. Hist. Bull., 31: 1-185.

Pollock, J. I., 1975. Field observations on Indri indri, a preliminary report. In: Lemur Biology, I. Tattersall \& R. W. Sussman (eds.), Plenum Press, New York, pp. 287-311. 
Richard, A. F., 1974. Intra-specific variation in the social organization and ecology of Propithecus verreauxi. Folia Primat., 22: 178-207.

Schuman, E. L. \& C. L. BRACE, 1954. Metric and morphologic variations in the dentition of the Liberian chimpanzee; comparisons with anthropoid and human dentitions. Hum. Biol, 26: 239-268.

Schwartz, J. H., 1974. Observations on the dentition of the Indriidae. Am. J. Phys. Anthrop., 41: 107-114.

Swindler, D. R., 1976. Dentition of Living Primates, Academic Press, New York.

TatTersall, I., 1971. Revision of the subfossil Indriidae. Folia Primat., 16: 257-269.

$\ldots$, 1973a. Cranial anatomy of Archeolemurinae (Lemuroidea, Primates). Anthrop. Pap. Amer. Mus. Nat. Hist., 52: 1-110.

, $1973 \mathrm{~b}$. Subfossil lemuroids and the "adaptive radiation" of the Malagasy lemurs. Trans. N. Y. Acad. Sci., 35: 314-324.

Van Valen, L., 1962. Growth fields in the dentition of Peromyscus. Evolution, 16: 272-277.

-Received September 29, 1977; Accepted December 10, 1977

Authors' Address: Philip D. Gingerich, Museum of Paleontology, University of Michigan, Ann Arbor, Michigan 48109, U.S.A.; Alan S. RYan, Department of Anthropology, University of Michigan, Ann Arbor, Michigan 48109, U.S.A. 